\title{
Les clefs de la crise ivoirienne
}

. Paris, Karthala, (« Disputatio »), 2011, 140 p., bibl.

\section{Christian Bouquet}

\section{CpenEdition}

\section{Journals}

Édition électronique

URL : http://journals.openedition.org/etudesafricaines/17860

DOI : $10.4000 /$ etudesafricaines. 17860

ISSN : $1777-5353$

Éditeur

Éditions de l'EHESS

\section{Édition imprimée}

Date de publication : 3 octobre 2014

ISSN : 0008-0055

\section{Référence électronique}

Christian Bouquet, «Les clefs de la crise ivoirienne», Cahiers d'études africaines [En ligne], 215 | 2014 mis en ligne le 02 octobre 2016, consulté le 24 septembre 2020. URL : http://journals.openedition.org/ etudesafricaines/17860 ; DOI : https://doi.org/10.4000/etudesafricaines.17860

Ce document a été généré automatiquement le 24 septembre 2020.

(c) Cahiers d'Études africaines 


\title{
Les clefs de la crise ivoirienne
}

. Paris, Karthala, (« Disputatio »), 2011, 140 p., bibl.

\author{
Christian Bouquet
}

\section{DOZoN, Jean-Pierre. - Les clefs de la crise ivoirienne. Paris, Karthala, (« Disputatio »), 2011, 140 p., bibl.}

1 Laurent Gbagbo a toujours affirmé que les Blancs - et singulièrement les Français - ne pouvaient rien comprendre à la Côte-d'Ivoire. Il aurait pu faire au moins une exception pour ceux qui ont vécu dans ce pays et qui lui ont consacré des années de recherches, notamment dans le domaine croisé de l'histoire et de l'anthropologie. Jean-Pierre Dozon échapperait alors à cet oukaze verbal, d'abord parce qu'il a soutenu sa thèse sur le groupe auquel appartient l'ancien chef de l'État ${ }^{20}$, et ensuite parce qu'il apporte dans ce petit volume des éléments d'explication relevant justement de ses anciennes recherches dans le pays.

2 Après une première partie qui reprend la trame historique du drame ivoirien - déjà publiée ailleurs - Jean-Pierre Dozon revient sur les origines coloniales du malaise, ainsi que sur des causes datant de la période Houphouët. Là se situe l'originalité de l'ouvrage: ses explications relèvent bien de l'anthropologie lorsqu'elles approfondissent le démembrement du tissu social, voire familial, sous l'effet de la crise économique, lorsqu'elles pointent la criminalisation montante, ou lorsqu'elles évoquent la diaspora de plus en plus volumineuse, notamment en France, devenue « l'arrière-scène » de la crise ivoirienne.

En utilisant le terme de "brutalisation » pour intituler sa première partie, l'auteur cherche à gommer l'image relativement soft que la Côte-d'Ivoire souhaitait donner d'elle-même à travers ses historiographes et ses hagiographes. Le sous-titre est fort, mais il est justifié par une violence qui n'a pas toujours été soulignée dans l'histoire, que ce soit pendant la conquête, pendant la colonisation ou au début de l'indépendance. Ensuite, bien sûr, il y a eu une exacerbation de cette violence, entretenue à la fois par la FESCI (Fédération des étudiants et scolaires de Côte-d'Ivoire) et par la délinquance nouvelle liée à la crise économique et à la pression 
démographique. À ce sujet, il parle de «contre culture» avec la bienveillance des anthropologues. Il ajoute la violence de la presse écrite, et l'on comprend mieux pourquoi les accords de Marcoussis recommandaient avec insistance une remise à plat de ce secteur, largement dévoyé depuis la libération de la parole. Il aurait pu préciser que l'OLPED (Observatoire de la liberté de la presse) avait été fort judicieusement créé au début des années 1990 justement pour éviter les dérives, mais en vain. Peut-être eût-il été également pertinent de s'étendre davantage sur la «crise morale » qui a marqué le pays depuis que la corruption a atteint des niveaux inégalés.

4 Un autre point fort de ce petit volume se trouve dans l'étude particulière - dont l'auteur est spécialiste - du repli sur les religions, notamment les religions prophétiques. On retrouve les préoccupations de l'auteur telles qu'elles avaient été traitées dès $1995^{21}$ et en $2008^{22}$. Il revient d'ailleurs à plusieurs reprises dans le corps de l'ouvrage sur cette thématique des nouvelles religiosités qui lui tient à cœur, sans pour autant rappeler que l'une des voies d'intrusion du néo-pentecôtisme se trouvait dans les camps de réfugiés du grand Ouest pendant les crises libérienne et sierra-léonaise (le Haut commissariat aux réfugiés n'ayant plus les moyens d'assurer les soins de santé et l'éducation, les Églises évangéliques s'étaient engouffrées dans la faille).

5 Enfin, il expose - il en a la légitimité - son point de vue sur l'ethnie, et donc sur «l'autochtonie politique» et sur la «crise d'altérité » qui ont imprimé une tache particulière à la crise ivoirienne. Soulignant que la Côte-d'Ivoire est un pays où se sont multipliées les monographies ethniques, il en dénonce l'instrumentalisation, sans dire qui en fut à l'origine: les colonisateurs, les politiques, ou les chercheurs? Naturellement, on attendait Jean-Pierre Dozon sur l'ivoirité : il en parle comme d'une doctrine d'extrême droite, et l'actualité le confirme puisque, dans la diaspora, certains partisans ivoiriens de Laurent Gbagbo soutiennent Marine Le Pen (laquelle avait d'ailleurs apporté son appui à l'ancien chef de l'État). Prudent, il ne va pas jusqu'à citer les noms des intellectuels ivoiriens qui ont formalisé cette doctrine, notamment l'expression "Ivoiriens de souche multiséculaire », et qui l'ont défendue. On ajoutera que la dérive du FPI (Front populaire ivoirien) dans ce domaine (et dans d'autres) a connu son aboutissement avec sa suspension de l'Internationale socialiste en mars 2011.

6 On se réjouit de la distance prise par l'auteur avec la grille de lecture obsolète de la «Françafrique » (il préfère l'expression «État franco-africain »). De même, il rappelle fort opportunément que, contrairement à beaucoup d'autres pays colonisés, la Côted'Ivoire attribua ses terres à des immigrés africains bien plus qu'à des colons européens, et là se trouve également l'une des clés de compréhension de l'histoire du pays.

7 Il demeure dans l'ouvrage quelques coquilles sans doute dues à une relecture trop rapide (il manque systématiquement un « $\mathrm{r}$ " à Mitterrand), de même que des approximations qui auraient pu être évitées. Ainsi la ligne de démarcation qui a coupé le pays en deux entre 2002 et 2007 ne passait pas par Yamoussoukro : la zone de nonfranchissement (dite aussi «zone de confiance») se situait plusieurs dizaines de kilomètres plus au Nord. De même, la formule « la terre est à celui qui la cultive » n'est pas un «slogan » d'Houphouët-Boigny, mais une réalité - une belle utopie - du droit foncier traditionnel dans la plus grande partie de l'Afrique subsaharienne. C'est d'ailleurs la cause principale des conflits d'usage dans toute la bande sahélienne du continent. Enfin, la baisse des salaires des enseignants décrétée par Ouattara quand il 
était le Premier ministre d'Houphouët était en fait un "raccrochage " à la grille imposée par le FMI ; le décalage entre " décrochés » et " raccrochés » fut d'ailleurs une spécificité ivoirienne, en même temps que l'une des causes de la tension sociale de toute une décennie.

8 Dans sa conclusion, l'auteur revient sur le rendez-vous plusieurs fois manqué de la Côte-d'Ivoire avec le panafricanisme. Ce rappel prend toute son importance dans l'actualité puisque Alassane Ouattara vient d'être élu à la présidence de la CEDEAO, et qu'il commence à peser dans la diplomatie africaine. On attendait la contribution de Jean-Pierre Dozon au décryptage de la crise ivoirienne. Son petit ouvrage répond parfaitement aux attentes.

\section{NOTES}

20. J.-P. Dozon, La société bété. Histoire d'une «ethnie» de Côte-d'Ivoire, Paris, Karthala, 1985.

21. J.-P. Dozon, La cause des prophètes. Politique et religion en Afrique contemporaine, Paris, Éditions du Seuil, 1995.

22. J.-P. Dozon, L'Afrique à Dieu et à Diable, Paris, Ellipses, 2008. 